\title{
Perventricular Device Closure of Perimembranous Ventricular Septal Defect in Pediatric Patients: Technical and Morphological Considerations
}

\author{
Da Zhu ${ }^{1}$ ChangPing Gan ${ }^{1}$ Xiao Li ${ }^{2}$ Qi An ${ }^{1}$ Shuhua Luo ${ }^{1}$ Hong Tang ${ }^{2}$ Yuan Feng ${ }^{2}$ Ke Lin ${ }^{1}$ \\ ${ }^{1}$ Department of Cardiovascular Surgery, West China Hospital, Sichuan \\ University, Chengdu, Sichuan, China \\ 2 Department of Cardiology, West China Hospital, Sichuan University, \\ Chengdu, Sichuan, China \\ Address for correspondence Ke Lin, MD, Department of Cardiovascular \\ Surgery, West China Hospital, Sichuan University, Chengdu, Sichuan \\ 610041, P.R. China (e-mail: Edwarddian@gmail.com).
}

Thorac Cardiovasc Surg 2013;61:300-306.

\begin{abstract}
Background We report our experience of using perventricular device closure (PVDC) in treating perimembranous ventricular septal defect (pm-VSD) with emphasis on technical and morphological considerations.

Method Thirty-one pediatric patients with pm-VSD who underwent successful PVDC were enrolled in this study. The pm-VSDs were divided into three different types (type I: tunnel shape; type II: with subaortic rim $<2 \mathrm{~mm}$; type III: membranous aneurysm formation). Four closure strategies were utilized, corresponding to the morphology of the pm-VSD.

Results Mean age of the patients was 2.1 years with mean VSD diameter $5.8 \mathrm{~mm}$. Seven patients had type I VSD, nine presented with type II, and 15 had type III. Twentytwo concentric and nine eccentric devices were used with mean device size $7.3 \mathrm{~mm}$. Complete closure was achieved in $97 \%$ of cases during follow-up. Procedure-induced

Keywords

- perventricular device closure

- ventricular septal defect

- pediatric patients tricuspid regurgitation (TR) was noted in nine patients at discharge; four resolved. Multivariable analysis showed that the procedure-induced TR was associated with the device size (odds ratio $=5.059 ; 95 \%$ confidence interval $=1.431-17.880$ ). Conclusion Different closure strategies allow for PVDC of various types of pm-VSDs in selected pediatric patients.
\end{abstract}

\section{Introduction}

Perimembranous ventricular septal defect (pm-VSD) is the most common congenital cardiac malformation. Open heart surgery with cardiopulmonary bypass (CPB) is the conventional treatment option for this type of defect. ${ }^{1,2}$ Interventional transcatheter device closure is a much less invasive technique with encouraging outcomes, ${ }^{3-5}$ but it is still a challenging technique especially in pediatric patients with limited vascular access. Perventricular device closure (PVDC) of pm-VSD has been described in several studies with encouraging results. ${ }^{6-8}$ This method provides another treatment option for pediatric patients with pm-VSD who are not eligible for transcatheter closure.

Pm-VSD often presents with different kinds of morphology and is also adjacent to several important structures such as the conduction bundle and aortic and tricuspid valves. ${ }^{9,10} \mathrm{~A}$ different management strategy is necessary during the PVDC procedure, corresponding to the unique anatomical structure of pm-VSD. ${ }^{11}$ We report our experience in PVDC of pm-VSD received

November 8, 2012

accepted after revision

January 18, 2013

published online

April 5, 2013 (c) 2013 Georg Thieme Verlag KG Stuttgart · New York
DOI http://dx.doi.org/ 10.1055/s-0033-1334997. ISSN 0171-6425. 
for pediatric patients, with emphasis on morphological and technical aspects of this procedure.

\section{Materials and Methods}

\section{Patient Selection}

This study was approved by the institutional review board of our hospital, and informed consent was obtained from patients' parents. Between July 2010 and March 2011, patients with isolated pm-VSD who underwent successful minimally invasive PVDC were enrolled in this study. Inclusion criteria were: (1) pm-VSD with significant left-to-right shunt (defined by echocardiogram, X-ray, or symptom: cardiomegaly on chest X-ray, left atrial enlargement-to-aorta ratio > 1.5; left ventricle [LV] enlargement; LV end-diastolic volume $>2$ + standard deviations [SDs] above the mean for the patient's age; symptom of frequent respiratory infections or New York Heart Association class > II) and (2) patients younger than 3.5 years, who were not eligible for transcatheter closure due to vascular access limitation. Exclusion criteria were: (1) VSD with bidirectional shunt; (2) pm-VSD larger than $12 \mathrm{~mm}$ or with poor margin that was not eligible for device closure; (3) multiple VSDs; (4) confirmed pulmonary hypertension (echocardiogram was used for initial screening then catheter exam was done if necessary to confirm the diagnosis; mean pulmonary pressure $>25 \mathrm{~mm} \mathrm{Hg}$ with Wood index $>3.0$ ); (5) associated with other congenital heart defects; (6) failure of PVDC due to technical challenges or immediate deviceinduced complication (such as more than mild degree valve insufficiency, obvious residual shunt, and complete atrioventricular conduction block [cAVB]) and conversion to open heart surgery was also excluded from this study.

\section{Device and Delivery System}

Hardware used for the study included a pm-VSD occluder device and its delivery system (Shanghai Shape Memory Alloy Company, SHSMA, China). Two different kinds of occluder devices were used: concentric and eccentric ( - Fig. 1). The flanges of the two ventricular disks in the concentric type

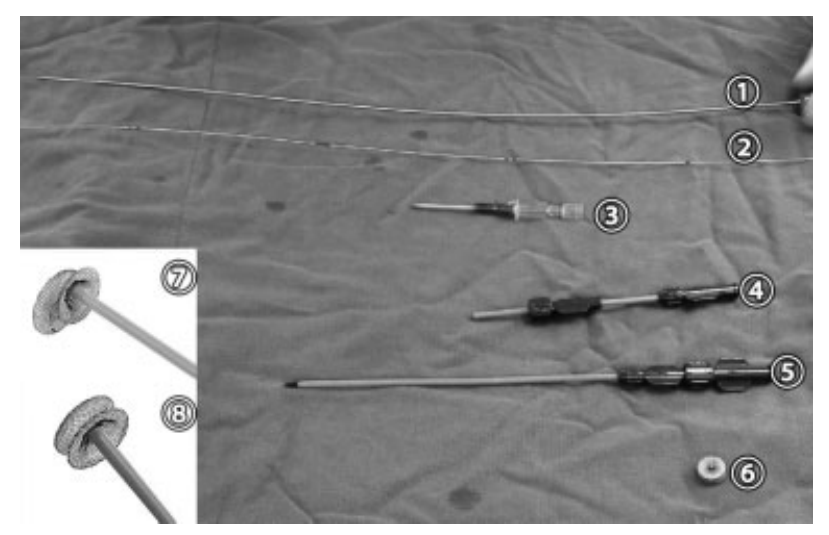

Fig. 1 Device and its delivery system. The delivery system consists of (1) a device cable, (2) a flexible guide wire, (3) a 20-gauge needle, (4) a device loading sheath, as well as (5) a double-layer delivery sheath. Two types of devices were used during the procedure: $(6,8)$ concentric and (7) eccentric devices. (Source: Shanghai Shape Memory Alloy Co. Ltd., China.) were 2-mm wide. The right ventricular (RV) disk of the eccentric type was the same as that of the concentric type, but the flange of the LV disk facing the aortic valve was $0.5-\mathrm{mm}$ wide or absent $(0 \mathrm{~mm})$ so as to prevent impingement of the aortic valve. The opposite flange at the same disk was $5-\mathrm{mm}$ long and had a metallic mark on its edge to indicate the orientation under transesophageal echocardiography (TEE). The entire delivery system consisted of a flexible guide wire and double-layer delivery sheath as well as a loading sheath (-Fig. 1).

\section{Preoperative Evaluation and PVDC Strategy Decision}

Pm-VSD was divided into three types according to its morphology visualized by TEE image. Type I was a tunnel-shaped pm-VSD, which was shrouded by tricuspid valve tissue ( - Fig. 2A and B). Type II was a pm-VSD with a short subaortic rim $(<2 \mathrm{~mm}$; - Fig. 3A-C). Type III was a pm-VSD with membranous aneurysm formation, including two subtypes: IIIa, large aneurysm formation ( $>9-10 \mathrm{~mm}$ in LV surface; - Fig. 4A and B), and IIIb, small aneurysm $(<7-8 \mathrm{~mm}$ in LV surface; $\boldsymbol{-}$ - Fig. $\mathbf{5 A}$ and $\mathbf{B})$.

Four different device closure strategies were chosen according to pm-VSD morphology. For type I pm-VSD, direct closure with a concentric device is recommended ( - Fig. 2 C). For type II pm-VSD, an eccentric device should be used to avoid the interface with the aortic valve ( - Fig. 3D). Two different strategies were designed for pm-VSD with aneurysm formation. For type IIla pm-VSD with large aneurysm formation, if the aneurysm structure was tough enough to support the device, we used the single device to close the orifice on the RV surface of the aneurysm (the device was inside the aneurysm; - Fig. 4C). For type IIIb pm-VSD, one device was used to cover all the membranous aneurysm (orifice on LV surface; - Fig. 5C).

\section{PVDC Procedure}

Step I: The free wall of the RV was exposed by a 2- to 4-cm lower partial median sternotomy, and systemic heparinization was achieved by giving $1 \mathrm{mg} / \mathrm{kg}$ heparin intravenously. TEE was performed to evaluate the pm-VSD. An occluder was chosen according to the location and diameter of the pm-VSD by consensus of the surgeon and the sonographer.

Step II: Under continuous TEE guidance, the surgeon gently depressed the RV-free wall with his index finger. A puncture site perpendicular to the plane of the VSD was selected. A pledgetted 4-0 Prolene pursestring suture was placed around the chosen puncture site.

Step III: A 20-gauge needle was inserted into the RV cavity under TEE guidance. A flexible guide wire was introduced into the RV through the needle and was maneuvered into the LV through the defect; the needle was then removed.

Step IV: A double-layer delivery sheath was advanced into the LV over the guide wire, and then the inner layer sheath and the guide wire were removed together, 


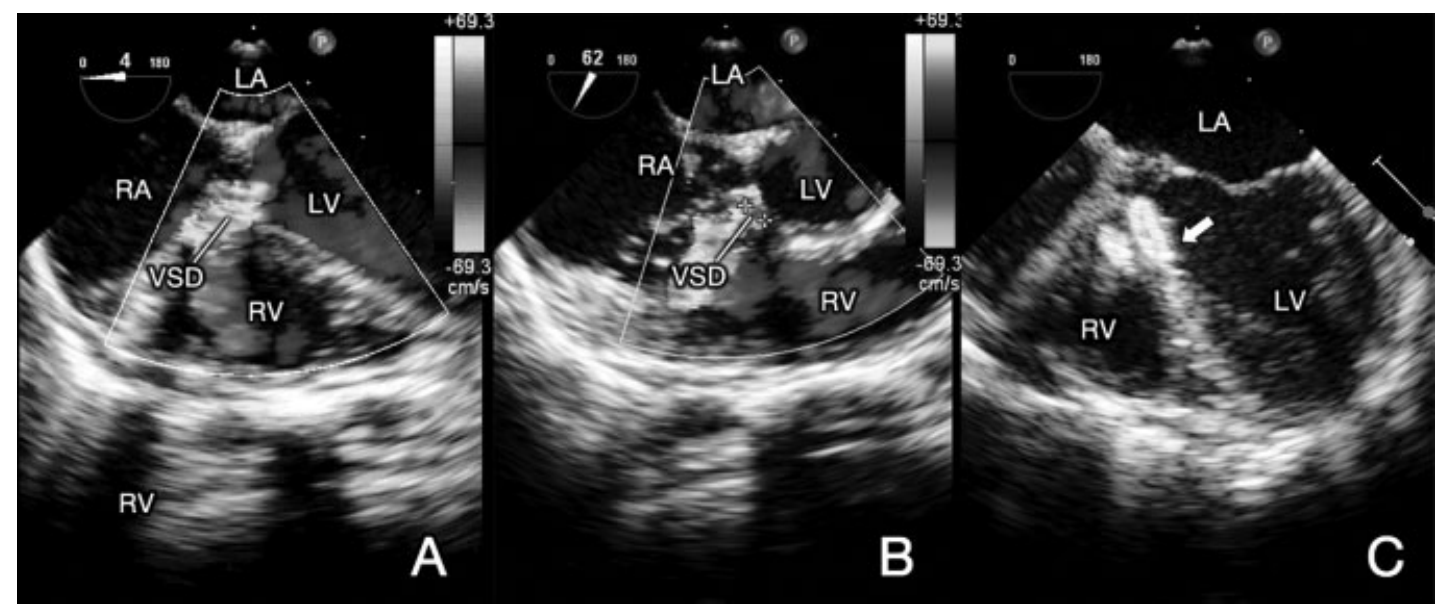

Fig. 2 Perventricular device closure strategy for type I pm-VSD. As shown in transesophageal echocardiography image, type I pm-VSD is a tunnel-shaped structure shrouded by tricuspid valve tissue. A single device 1 to $2 \mathrm{~mm}$ larger than the VSD diameter can be used for direct closure of this type of VSD. LA, left atrium; LV, left ventricle; RA, right atrium; RV, right ventricle; pm-VSD, perimembranous ventricular septal defect.

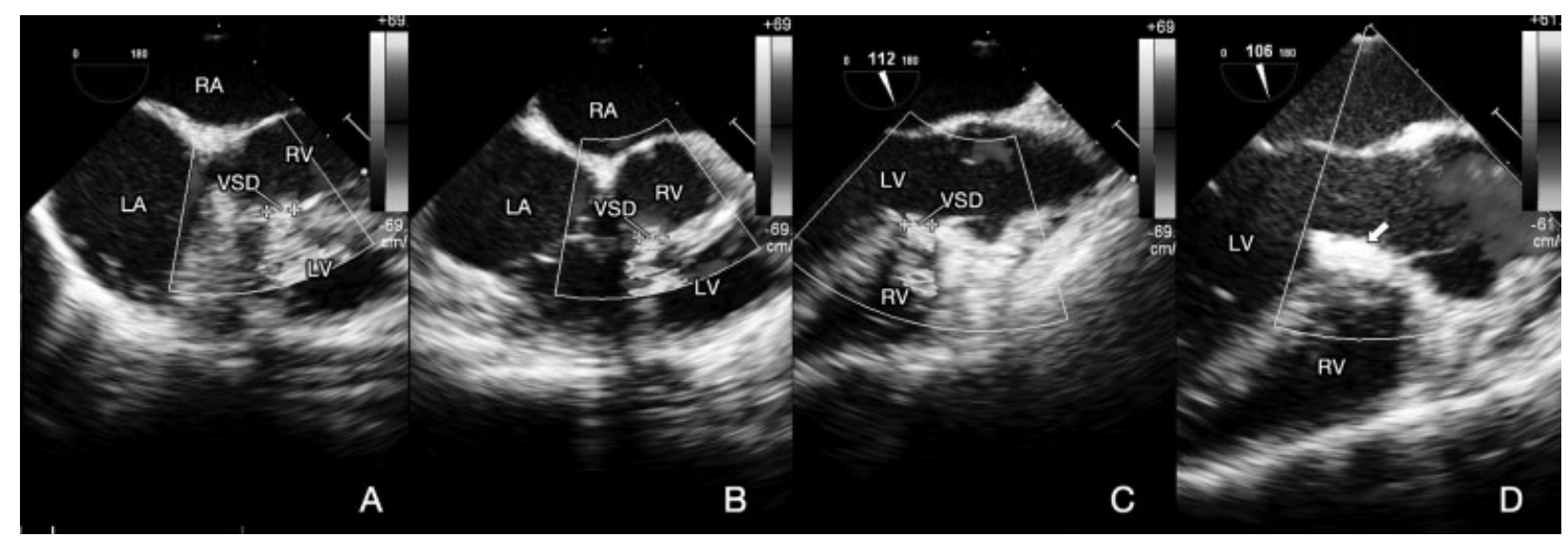

Fig. 3 Perventricular device closure strategy for type II pm-VSD. Type II pm-VSD is associated with a short subaortic rim $(<2 \mathrm{~mm})$ as shown in transesophageal echocardiography $(A-C)$. An eccentric device should be used to close the VSD while avoiding the interface with aortic valve (D). LA, left atrium; LV, left ventricle; RA, right atrium; RV, right ventricle; pm-VSD, perimembranous ventricular septal defect.

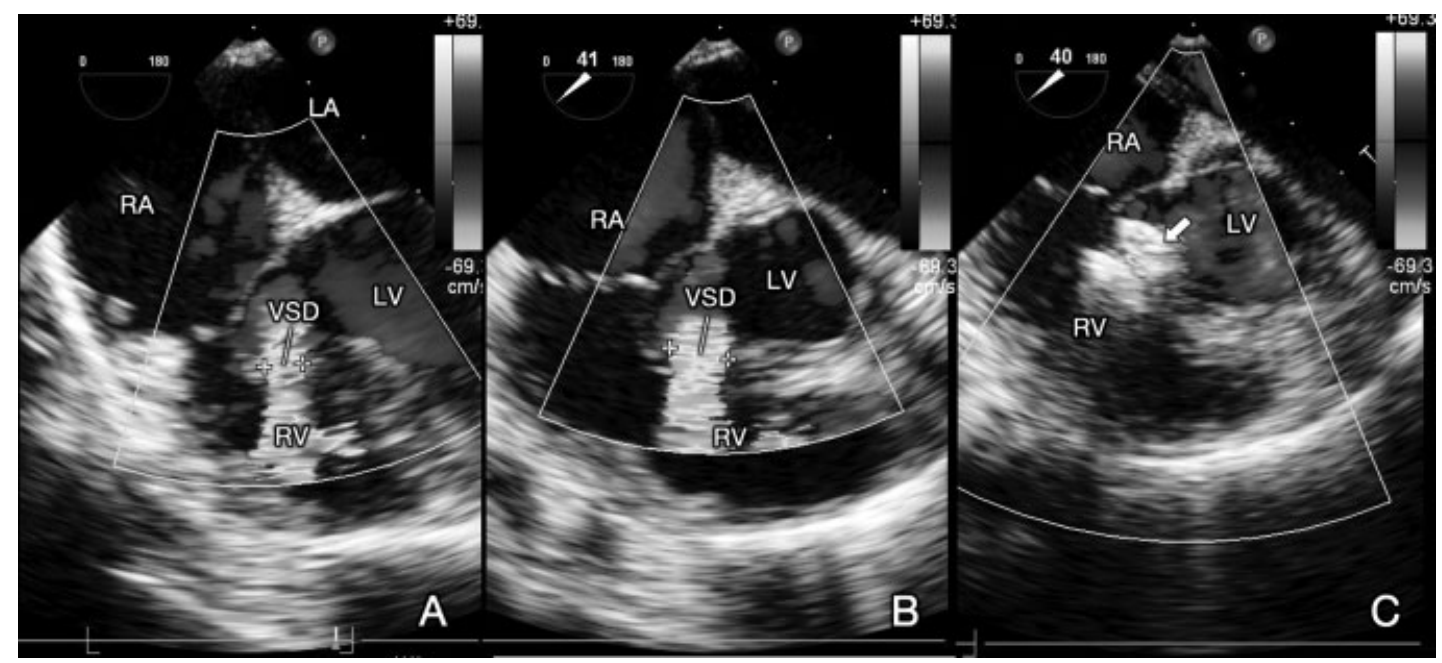

Fig. 4 Perventricular device closure strategy for type IIla pm-VSD. Type IIla pm-VSD is defined as having formation of a large aneurysm, more than $10 \mathrm{~cm}$, with a large orifice on the LV side, with the aneurysm tissue itself being stiff (A, B). For this type of VSD, one small device can be used to close the orifice on the RV side of the aneurysm to achieve complete closure (C). LA, left atrium; LV, left ventricle; RA, right atrium; RV, right ventricle; pm-VSD, perimembranous ventricular septal defect. 


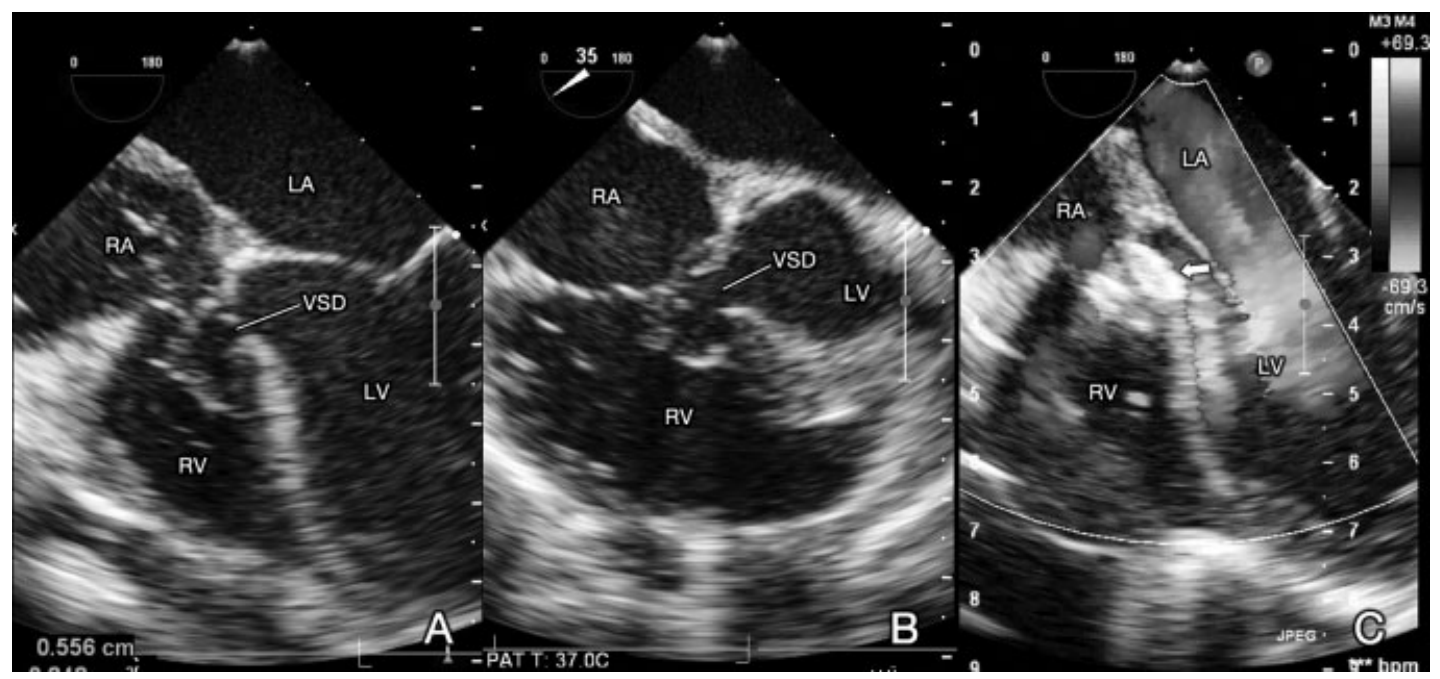

Fig. 5 Perventricular device closure strategy for type IIIb pm-VSD. Type IIIb pm-VSD is associated with formation of a small aneurysm, less than 7 to $8 \mathrm{~mm}$, on the LV surface (A, B). One device can be used to close the whole pm-VSD for this type of VSD (C). LA, left atrium; LV, left ventricle; RA, right atrium; RV, right ventricle; pm-VSD, perimembranous ventricular septal defect.

leaving the outer delivery sheath in position to guide further delivery. During the procedure, the purse-string suture was tightened to prevent excessive blood loss.

Step V: The previously prepared loader sheath was then connected to the free end of the delivery sheath. Under the TEE guidance, the LV disk was deployed first by pushing the cable forward while holding the delivery sheath still; once the expanded LV disk was visualized by TEE, the cable and the delivery sheath were gently pulled back until the disk was pulled snugly against the left side of the ventricular septum. The waist of the device and the RV disk were then deployed. As for VSD with a short aortic rim, the marker in the eccentric device should be adjusted to the opposite side of the aortic valve by rotating the delivery sheath just after the deployment of the left-sided disk.

Step VI: If no complication such as device malposition, obvious residual shunt, or device-induced new valve regurgitation was detected by TEE, the loading sheath and the carrier cable were withdrawn simultaneously, and the device was then released. All patients received aspirin ( $3 \mathrm{mg} / \mathrm{kg}$ per day) orally, starting on first postoperative day and continuing for 6 months.

\section{Outcome Measurement}

Standard 12-lead electrocardiogram (EKG), chest radiograph, and transthoracic echocardiogram (TTE) were performed routinely on postoperative day 2 or 3 , before discharge, and at 6 and 12 months during follow-up. Twenty-four-hour electrocardiographic Holter monitoring was performed if necessary.

\section{Statistical Analysis}

Data are expressed as percentage for nominal variables and mean \pm SD (range) for continuous variables. SPSS 16.0 for
Windows (SPSS Inc., Chicago, Illinois, USA) was used for statistical analysis. Residual shunts, tricuspid regurgitation (TR), aortic regurgitation (AR), cAVB, and incomplete right bundle branch block were analyzed as dependent outcome variables. Gender, age, weight, subaortic rim, VSD diameter, ratio of VSD diameters to device size and to patient's weight separately as well as the ratio of the device size to the patient's weight were analyzed as independent variables.

Univariate analysis was performed using Student's $t$-test and chi-square test or Fisher exact test. Multiple logistic regression analysis was performed to evaluate the risk factor for early or midterm complications. Independent variables with a $p$ value less than 0.2 in the univariate analysis and factors that were considered of clinical relevance were all included in the multivariable analysis. Odds ratios and 95\% confidence intervals (CIs) were calculated. $p<0.05$ was considered statistically significant in multivariable analysis.

\section{Result}

\section{Patients' Basic Information}

During the study period, a total of 40 patients with pm-VSD underwent PVDC in our hospital. Among them, 31 pediatric patients with successful PVDC of pm-VSD were enrolled in this study with mean age $2.1 \pm 0.9$ years (range $0.4-3.5$ ) and mean weight $12.7 \pm 3.4 \mathrm{~kg}$ (range 5.5-19; - Table 1). In other nine patients, PVDC failed due to difficulty in building the track (three patients), more than mild degree of TR (two patients), AR (two patients), and CAVB (one patient) immediately after device deployment, as well as obvious residual shunt due to partial device detachment during the procedure (one patient).

\section{Intraoperative Data}

Mean VSD size among 31 patients was $5.8 \pm 1.7 \mathrm{~mm}$ (range 3-10.5 mm) in TTE and $5.5 \pm 1.8$ (range 3.5-11.1) in TEE. TEE confirmed with type I VSD (tunnel-shaped or shrouded by 
Table 1 Perioperative data of 31 patients who underwent perventricular closure

\begin{tabular}{|l|l|}
\hline Category & \multicolumn{2}{l|}{} \\
\hline Mean age $(\mathrm{y})$ & $2.1 \pm 0.9$ (range 0.4-3.5) \\
\hline Mean body weight $(\mathrm{kg})$ & $12.7 \pm 3.4$ (range 5.5-19) \\
\hline Mean VSD diameter in TTE $(\mathrm{mm})$ & $5.8 \pm 1.7$ (range 3.7-10.5) \\
\hline Mean VSD diameter in TEE $(\mathrm{mm})$ & $5.5 \pm 1.8$ (range 3.5-11.1) \\
\hline Preoperative valve regurgitation & 12 trivial, 3 mild \\
\hline Tricuspid regurgitation & 1 trivial \\
\hline Aortic regurgitation & 1 trivial, 1 mild \\
\hline Pulmonary regurgitation & \multicolumn{2}{|l}{} \\
\hline Type of VSD (n) & 7 \\
\hline Type I & 9 \\
\hline Type II & 10 \\
\hline Type IIla & 5 \\
\hline Type IIIb & $22 / 9$ \\
\hline Device type (concentric/eccentric) & $7.3 \pm 2.0$ (range 4-12) \\
\hline Mean device size & $(4 / 31) 13 \%$ \\
\hline Transfusion rate & $1.2 \pm 0.2(1-1.5)$ \\
\hline Mean follow up time (y) &
\end{tabular}

Abbreviations: TEE, transesophageal echocardiogram; TTE, transthoracic echocardiogram; VSD, ventricular septal defect.

extensive tricuspid valve tissue) in 7 patients, type II VSD (subaortic rim $<2 \mathrm{~mm}$ ) in 9 patients, and type III (membranous aneurysm formation) in 15 . Twenty-two concentric and nine eccentric devices were used during the procedure with mean device size $7.3 \pm 2.0 \mathrm{~mm}$ (range 4-12 mm). Intraoperative data are shown in -Table $\mathbf{1}$.

\section{Postoperative Complications}

There was no mortality or severe complication such as device embolism, malignant arrhythmia, as well as congestive heart failure during hospital stay among study cohort (- Table 2). Complete closure was achieved in $84 \%$ immediately after device deployment and $90 \%$ at discharge with all residual shunts less than $2 \mathrm{~mm}$. Procedure-induced TR was noted in eight patients (five trivial and three mild) just after device deployment; at discharge, eight patients were found to have trivial TR and one had mild TR. Device-induced trivial AR was noted in one patient with type II pm-VSD at discharge. Another patient was found to have device-induced trivial pulmonary regurgitation just after device deployment, which resolved spontaneously at discharge. One patient developed second-degree atrioventricular conduction block (Mobitz I) after device deployment, which was resolved with intravenous steroids in the first postoperative day.

\section{Follow-up Data}

All 31 patients finished at least 1 year of follow-up with mean follow-up of $1.2 \pm 0.2$ years (range $1-1.5$ ). There was no mortality or severe complication such as device embolism or congestive heart failure during follow-up. Residual shunt $(<2 \mathrm{~mm})$ was noted in one patient during follow-up, and trivial TR was noted in five patients through TEE. One patient

Table 2 Complications of the 31 patients with successful perventricular device closure

\begin{tabular}{|l|l|l|l|}
\hline \multirow{2}{*}{ Category } & \multicolumn{3}{|c|}{ Postclosure } \\
\cline { 2 - 4 } & In OR (TEE) & Discharge (TTE) & Follow-up (TTE) \\
\hline Residual shunt & $5(<2 \mathrm{~mm})$ & $3(<2 \mathrm{~mm})$ & $1(<2 \mathrm{~mm})$ \\
\hline Procedure-related valve regurgitation & 5 trivial/3 mild & 8 trivial/1 mild & 5 trivial \\
\hline Tricuspid regurgitation & 1 trivial & 1 trivial & 0 \\
\hline Aortic regurgitation & 1 trivial & 0 & 0 \\
\hline Pulmonary regurgitation & 1 (2nd-degree AVB) & 0 & 1 complete AVB \\
\hline AVB
\end{tabular}

Abbreviations: AVB, atrioventricular conduction block; OR, operating room; TEE, transesophageal echocardiogram; TTE, transthoracic echocardiogram. 
suffered from transient syncope 8 months after the procedure; Holter EKG confirmed complete atrioventricular conduction block and an endocardial ventricular pacemaker was then implanted. The patient recovered uneventfully. Data are shown in - Table 2.

\section{Multivariable Analysis}

Univariate analysis showed the postoperative residual shunt confirmed by TEE in the operating room was associated with VSD diameter $(p=0.045)$. However, multiple logistic regressions including two other clinically relevant factors (device size as well as device size and weight) showed no relationship between these variables and residual shunt. Univariate analysis suggested that the procedure-induced TR confirmed by TEE in the operating room was associated with VSD size ( $p=0.029)$, device size $(p=0.008)$, as well as device size and weight $(p=0.094)$. Multiple logistic regressions revealed TR in the operating room was associated with device size (odds ratio $=2.731 ; 95 \% \mathrm{CI}=1.086-6.868$ ). The same relationship was also duplicated between the device size and TR confirmed by TTE in discharge after multivariable analysis (odds ratio $=5.059 ; 95 \% \mathrm{CI}=1.431-17.880$ ).

\section{Discussion}

Our results showed that PVDC of pm-VSD with different sizes and morphologies is a feasible, safe, and effective method for pediatric patients. It has the advantage of avoiding CPB during conventional open surgical repair while providing another minimally invasive treatment option for pediatric patients who were not eligible for transcatheter closure due to vascular limitations. ${ }^{6,7,12-14}$ Also, due to the direct access of this single-shunt technique, building the delivery track and adjusting the position of the device during deployment will be much easier in comparison with a transcatheter approach, especially for pediatric patients. This technique is so far widely accepted in China and was also reported by a German surgeon with encouraging initial results. $6,7,12-15$

However, until now no study has discussed the issue concerning the technical and morphological considerations of PVDC closure of pm-VSD. In our study, four different device closure strategies were used corresponding to the unique anatomical structures of pm-VSD. The purpose of this was to achieve complete closure while minimizing the potential interface between device and the important cardiac structures. For pm-VSD with a small aneurysm, using one concentric device to cover the aneurysm seems feasible. For pm-VSD with large aneurysm formation, using one device to cover all the aneurysms could be associated with mushrooming of the device. The central waist of the device is likely to be squeezed by the exit hole on the right side as well as the tricuspid valve or conduction system, ${ }^{11}$ which may cause subsequent residual shunt and device malposition. Using a relatively small device to close the exit hole of the aneurysm is a more reliable way to achieve complete closure of pm-VSD in pediatric patients. However, according to our experience, for multifenestrated aneurysm or a big aneurysm with a thin and fragile aneurysm wall, perventricular closure is not recommended due to the potential risk of obvious residual shunt and device embolism. Using two devices to occlude multiple shunts in pm-VSD is also prohibited because of the risk of conduction block. For patients with no aneurysm formations, determination of the VSD size and selection of a device 1 to $2 \mathrm{~mm}$ larger than the defect is more straightforward. When a short subaortic rim $(<2 \mathrm{~mm})$ was noted during the TEE exam, an eccentric device was required with the zero margin side toward the valve to minimize the interface with aortic valve. During the device deployment process, TEE is crucial to make sure the right position of the eccentric device is achieved.

The occurrence of cAVB is the most serious complication related to device closure of $\mathrm{VSD},{ }^{16-20}$ which may lead to sudden cardiac death. During a transcatheter approach, the catheter must go through the tricuspid valve, which will cause unavoidable frictional rubbing with the conduction system. In contrast, the PVDC technique could avoid this phenomenon through its direct access and hence minimize the initial inflammatory response and subsequent scar formation within the conduction tissue. However, although the occurrence rate of CAVB reported in several studies was relatively lower in perventricular closure (3.2\% in this study) comparison with the transcatheter approach among pediatric patients, $6,7,12-18$ the potential risk of CAVB should still be highlighted for PVDC, especially for pediatric patients.

Because of the vicinity of pm-VSDs and the tricuspid valve, it has been hypothesized that a device in this region may interfere with valve function. ${ }^{11,12}$ Studies have shown that the RV disk does remain close to the tricuspid valve after deployment. ${ }^{16-20}$ In our study, nine patients presented with procedure-induced trivial to mild TR at discharge, and multivariate analysis showed it was associated with the device size. Although tricuspid insufficiency is not an issue in the short term and the septal leaflet of the tricuspid valve is not important for valve function, ${ }^{21}$ caution is still needed for the long-term outcome for pediatric patients. According to our experience, a device larger than $10 \mathrm{~mm}$ was not recommended in a pediatric patient less than 3.5 years old.

As one of the alternative methods to conventional open surgical repair, minimally invasive PVDC is now only indicated in selected patients with pm-VSD. However, evidence on the criteria of eligibility of PVDC for pediatric patient with pm-VSD is still limited. According to our initial experience, pm-VSDs larger than $10 \mathrm{~mm}$, with poor margin, multiple holes, or giant fragile aneurysm formation, were not all suitable for PVDC. Meanwhile, this procedure should also be abandoned if technical challenge or immediate device-induced complication such as obvious valve regurgitation, residual shunt, or complete heart block is noted during the procedure.

\section{Limitation}

This is a relatively small observational study with limited follow-up; also, no angiography was performed during preoperative evaluation and therefore no hemodynamic data are available. Although we introduced the technical and morphological consideration in perventricular closure of 
pm-VSD, a large-scale study with longer follow-up is still necessary to validate this result.

\section{Conclusion}

PVDC is an effective treatment option for pediatric patients with pm-VSD. A different closure strategy is necessary during PVDC of pm-VSD presenting with different morphology.

\section{Note}

This article was presented at the 26th EACTS Annual Meeting, Barcelona, Spain.

\section{References}

1 Gessler P, Schmitt B, Prètre R, Latal B. Inflammatory response and neurodevelopmental outcome after open-heart surgery in children. Pediatr Cardiol 2009;30(3):301-305

2 Limperopoulos C, Majnemer A, Shevell MI, et al. Predictors of developmental disabilities after open heart surgery in young children with congenital heart defects. J Pediatr 2002;141(1): 51-58

3 Knauth AL, Lock JE, Perry SB, et al. Transcatheter device closure of congenital and postoperative residual ventricular septal defects. Circulation 2004;110(5):501-507

4 Hijazi ZM, Hakim F, Haweleh AA, et al. Catheter closure of perimembranous ventricular septal defects using the new Amplatzer membranous VSD occluder: initial clinical experience. Catheter Cardiovasc Interv 2002;56(4):508-515

5 Carminati M, Butera G, Chessa M, Drago M, Negura D, Piazza L. Transcatheter closure of congenital ventricular septal defect with Amplatzer septal occluders. Am J Cardiol 2005;96(12A):52L-58L

6 Tao K, Lin K, Shi Y, et al. Perventricular device closure of perimembranous ventricular septal defects in 61 young children: early and midterm follow-up results. J Thorac Cardiovasc Surg 2010; 140(4):864-870

7 Xing Q, Pan S, An Q et al. Minimally invasive perventricular device closure of perimembranous ventricular septal defect without cardiopulmonary bypass: multicenter experience and mid-term follow-up. J Thorac Cardiovasc Surg 2010;139(6):1409-1415

8 Bacha EA, Cao QL, Galantowicz ME, et al. Multicenter experience with perventricular device closure of muscular ventricular septal defects. Pediatr Cardiol 2005;26(2):169-175
9 Ho SY, McCarthy KP, Rigby ML. Morphology of perimembranous ventricular septal defects: implications for transcatheter device closure. J Interv Cardiol 2004;17(2):99-108

10 Penny DJ, Vick GW III. Ventricular septal defect. Lancet 2011; 377(9771):1103-1112

11 Pedra CA, Pedra SR, Esteves CA, et al. Percutaneous closure of perimembranous ventricular septal defects with the Amplatzer device: technical and morphological considerations. Catheter Cardiovasc Interv 2004;61(3):403-410

12 Xing Q, Wu Q, Pan S, Ren Y, Wan H. Transthoracic device closure of ventricular septal defects without cardiopulmonary bypass: experience in infants weighting less than $8 \mathrm{~kg}$. Eur J Cardiothorac Surg 2011;40(3):591-597

13 Amin Z, Gu X, Berry JM, Titus JL, Gidding SS, Rocchini AP. Perventricular [correction of Periventricular] closure of ventricular septal defects without cardiopulmonary bypass. Ann Thorac Surg 1999;68(1):149-153, discussion 153-154

14 Schreiber C, Vogt M, Kühn A, et al. Periventricular closure of a perimembranous VSD: treatment option in selected patients. Thorac Cardiovasc Surg 2012;60(1):78-80

15 Pan S, Xing Q, Cao Q, et al. Perventricular device closure of doubly committed subarterial ventral septal defect through left anterior minithoracotomy on beating hearts. Ann Thorac Surg 2012; 94(6):2070-2075

16 Butera G, Carminati M, Chessa M, et al. Transcatheter closure of perimembranous ventricular septal defects: early and long-term results. J Am Coll Cardiol 2007;50(12):1189-1195

17 Carminati M, Butera G, Chessa $M$, et al; Investigators of the European VSD Registry. Transcatheter closure of congenital ventricular septal defects: results of the European Registry. Eur Heart J 2007;28(19):2361-2368

18 Butera G, Carminati M, Chessa M, et al. Percutaneous closure of ventricular septal defects in children aged $<12$ : early and midterm results. Eur Heart J 2006;27(23):2889-2895

19 Rigby ML, Redington AN. Primary transcatheter umbrella closure of perimembranous ventricular septal defect. Br Heart J 1994; 72(4):368-371

20 Holzer R, de Giovanni J, Walsh KP, et al. Transcatheter closure of perimembranous ventricular septal defects using the Amplatzer membranous VSD occluder: immediate and midterm results of an international registry. Catheter Cardiovasc Interv 2006;68(4): 620-628

$21 \mathrm{Gu}$ X, Han YM, Titus JL, et al. Transcatheter closure of membranous ventricular septal defects with a new nitinol prosthesis in a natural swine model. Catheter Cardiovasc Interv 2000;50(4): 502-509 